\title{
Safety of Intradialytic Bamlanivimab/ Etesevimab Administration in Two COVID-19 Dialysis Outpatients
}

\author{
Lorenzo Gasperoni Chiara Abenavoli Gabriele Donati Anna Scrivo \\ Fulvia Zappulo Alessandra Cingolani Lilio Hu Gaetano La Manna \\ Nephrology Dialysis and Renal Transplantation Unit, IRCCS Azienda Ospedaliero Universitaria di Bologna, \\ Department of Experimental, Diagnostic and Speciality Medicine (DIMES), University of Bologna, Bologna, Italy
}

\section{Keywords}

Bamlanivimab · Etesevimab · SARS-CoV-2 $\cdot$ Hemodialysis · COVID-19

\begin{abstract}
Chronic hemodialysis patients are at high risk of severe COVID-19 disease and death related to the infection. Anti-spike monoclonal antibodies administration reduces risk of disease progression and hospitalization in high-risk subjects but no clear data on end-stage renal disease are available. We report 2 cases of Bamlanivimab/Etesevimab administration to two not hospitalized chronic hemodialysis patients with SARS-CoV2 infection. Since they are large molecules (human immunoglobulin G1) with molecular weight of $146,000 \mathrm{Da}$, administration was conducted during the second hour of the dialysis session with no adverse reaction. Conclusions: Intradialytic administration of Bamlanivimab/ Etesevimab could be considered safe and may allow adequate clinical observation time without hospital-stay prolongation.

C 2022 S. Karger AG, Basel
\end{abstract}

\section{Introduction}

Patients with end-stage renal disease (ESRD) requiring hemodialysis are one of the most vulnerable categories to develop severe COVID-19 disease [1], intensive care stay, and death related to COVID-19 infection [2]. Bamlanivimab (LY3819253 or LY-CoV555) and Etesevimab (LY3832479 or LY-CoV016) are anti-spike neutralizing monoclonal antibodies that block SARS-CoV-2 entry into cells $[3,4]$.

BLAZE-1 trial demonstrated the efficacy of Bamlanivimab alone and of the combination Bamlanivimab/ Etesevimab in reducing viral load and hospitalization in patients with mild or moderate COVID-19 infection, who were not hospitalized [5]. Renal failure was not an exclusion criterion but no data on renal function was reported in the results.

Since on February 2021, the Italian Medicines Agency (AIFA) authorized their administration in COVID-19 nonhospitalized patients aged 12 or older that do not require supplemental oxygen for COVID-19 and who are at high risk of progressing to severe infection we administered a single dose of Ely Lilly's monoclonal antibody combination therapy to two not hospitalized chronic hemodialysis patients. karger@karger.com www.karger.com/bpu (c) 2022 S. Karger AG, Basel

Karger
Correspondence to:

Gaetano La Manna, gaetano.lamanna@unibo.it 
Table 1. Clinical history, laboratory analysis, and patients' outcome

\begin{tabular}{|c|c|c|}
\hline & Patient 1 & Patient 2 \\
\hline Age, years/sex & $81 / \mathrm{male}$ & 77/male \\
\hline Dialysis vintage, months & 5 & 21 \\
\hline Charlson score & 11 & 6 \\
\hline Vascular access & Femoral tunneled CVC & Distal arteriovenous fistula \\
\hline Comorbidities & HFrEF, DM, AF, obesity & Surgically treated colorectal cancer, MGUS \\
\hline Dialysis technique & OLHDF & OLHDF \\
\hline Dialysis membrane & PMMA & PMMA \\
\hline Dialysis sessions during infection period, $n$ & 3 & 4 \\
\hline $\mathrm{Qb}, \mathrm{mL} / \mathrm{min}, \operatorname{median}(\min ; \max )$ & $200(200 ; 250)$ & $300(250 ; 300)$ \\
\hline Convective volume, L, median (min-max) & $14.5(14.2-14.9)$ & $15.9(14.7-16.9)$ \\
\hline Fluid removal per session, L, median (min-max) & $0.5(0.2-0.9)$ & $2.8(2.7-2.9)$ \\
\hline \multicolumn{3}{|l|}{ Clinical and laboratory parameters at diagnosis } \\
\hline Blood pressure, $\mathrm{mm} \mathrm{Hg}$ & $110 / 60$ & $140 / 70$ \\
\hline Symptoms & Cough/asthenia & Asthenia \\
\hline $\mathrm{SpO}_{2} \%$, room air & 99 & 97 \\
\hline $\mathrm{P} / \mathrm{F}$ ratio & 490 & 476 \\
\hline IL-6, pg/mL & 146.6 & 23.7 \\
\hline $\mathrm{PCR}, \mathrm{mg} / \mathrm{dL}$ & 13.99 & 2.42 \\
\hline $\mathrm{PCT}, \mathrm{ng} / \mathrm{mL}$ & 0.6 & 0.3 \\
\hline Infection duration, days & 9 (until death) & 11 \\
\hline Follow-up, days & 9 & 70 \\
\hline IL6 RR \%, median (min; max) & $-34(-71 ; 52)$ & $-20(-174 ;-90)$ \\
\hline Outcome & Death & $\begin{array}{l}\text { Infection recovery and symptoms resolution } 24 \mathrm{~h} \text { after } \\
\text { administration }\end{array}$ \\
\hline
\end{tabular}

HFrRF, heart failure with reduced ejection fraction; DM, diabetes mellitus, AF, atrial fibrillation; OLHDF, on-line hemodiafiltraton; PMMA, polymethylmethacrylate; MGUS, monoclonal gammopathy of uncertain significance.

\section{Case 1}

The first patient was an 81-year-old male with ESRD due to diabetic kidney disease on hemodialysis with a four times weekly schedule through a tunneled femoral catheter. Comorbidities were chronic heart failure, pace-maker for advanced atrioventricular block, atrial fibrillation, and metabolic syndrome.

Nasopharyngeal swab was performed because of contact with a COVID patient and at diagnosis mild cough and asthenia were present. Symptoms started on day 0 and he was tested the same day. Results were positive on day 1, and he received Bamlanivimab/ Etesevimab on day 2 during the first dialysis session after diagnosis. No adverse reactions were recorded. The day after he experienced rapid clinical worsening with due to severe COVID-19 pneumonia. He was admitted to our hospital and died after 9 days.

\section{Case 2}

The second patient was a 77-year-old male with ESRD due to diabetic kidney disease on hemodialysis with a twice weekly schedule through an arteriovenous fistula. He had total colectomy for carcinoma and monoclonal gammopathy of uncertain significance.

Nasopharyngeal swab was performed because of contact with a positive family member and asthenia was the only symptoms he suffered. Patient was tested on day 0 , he received the positive result the same day, symptoms started on day 4 and he received Bamlanivimab/Etesevimab on day 7 during the first dialysis session after symptoms onset. No adverse reactions were recorded. He remained clinically stable and symptoms resolved $24 \mathrm{~h}$ after administration and then recovered from infection after 11 days. Patients' clinical and laboratory features are shown in Table 1.

\section{Discussion}

Since etesevimab and bamlanivimab are large protein molecules (human immunoglobulin G1) with molecular weights of $146,000 \mathrm{Da}$, administration was conducted during the second hour of the hemodialysis session allowing an adequate clinical observation time without hospital-stay prolongation. The dose administered was 2,100 $\mathrm{mg}$ (700 mg $+1,400 \mathrm{mg}$ of Bamlanivimab and Etesevimab, respectively).

Both patients underwent on-line hemodiafiltration with a high-flux PMMA dialyzer with a surface area of 2.1 $\mathrm{m}^{2}$, a membrane cut-off value of 20,000 Da and an ultrafiltration coefficient (Kuf) of $43 \mathrm{~mL} / \mathrm{h} / \mathrm{mm} \mathrm{Hg}$. They had 
opposite outcomes. In the first case, we hypothesize that comorbidity burden, bacterial infection, and higher inflammatory response at presentation (see IL6 levels) played a role. To our knowledge, this is the first report of bamlanivimab/etesevimab administration in nonhospitalized chronic hemodialysis patients.

\section{Conclusions}

In our experience with 2 patients, administration of bamlanivimab/etesevimab during hemodialysis was not associated with any immediate infusion reactions.

\section{Statement of Ethics}

According to our local ethical committee (Comitato Etico Area Vasta Emilia Centro) case reports do not need specific approval. Written informed consent was obtained from the patients for publication of this case report and any accompanying images.

\section{Conflict of Interest Statement}

The authors have no conflict of interest to declare.

\section{Funding Sources}

This research received no external funding.

\section{Author Contributions}

The followings are the authors' contributions: conceptualization, writing, original draft preparation: L.G., C.A., G.D.; data collection: C.A., L.H., A.C.; methodology on laboratory: L.G., C.A., A.S., F.Z.; main supervision: L.G., C.A., G.L.M. All authors have read and agreed to the published version of the manuscript.

\section{Data Availability Statement}

All data generated during this study are included in this case report.
References

Bamlanivimab/Etesevimab in 2 COVID-19 Dialysis Patients
1 Hsu CM, Weiner DE. COVID-19 in dialysis patients: outlasting and outsmarting a pandemic. Kidney Int. 2020;98:1402-4.

2 Xiong F, Tang H, Liu L, Tu C, Tian J, Lei C, et al. Clinical characteristics of and medical interventions for COVID-19 in hemodialysis patients in Wuhan, China. J Am Soc Nephrol. 2020;31(7):1387-97.

3 Hiranoand T, Murakami M. COVID-19: a new virus, but a familiar receptor and cytokine release syndrome. Immunity. 2020 May; 52(5):731-3.
4 Gottlieb RL, Nirula A, Chen P, Boscia J, Heller B, Morris J, et al. Effect of bamlanivimab as monotherapy or in combination with etesevimab on viral load in patients with mild to moderate COVID-19: a randomized clinical trial. JAMA. 2021;325(7):632-44.

5 Chen P, Nirula A, Heller B, Gottlieb RL, Boscia J, Morris J, et al. SARS-CoV-2 neutralizing antibody LY-CoV555 in outpatients with covid. N Engl J Med. 2021;384:229-37. 